\title{
FIDEDIGNIDADE DA ESTRUTURA FATORIAL DA ESCALA MULTIDIMENSIONAL DE REATIVIDADE INTERPESSOAL (EMRI)
}

\author{
Nilton S. Formiga \\ Faculdade Mauricio De Nassau - João Pessoa, PB \\ Marseilly Carvalho Oliveira Rocha \\ Amanda de Souza Santos Pinto \\ Daniela Aparecida dos Reis \\ Sônia Maria da Silva Costa \\ Centro Universitário Do Cerrado - Unicerp - Patrocínio, MG \\ Jamila Leime \\ Universidade Federal Da Paraiba - UFBP - João Pessoa, PB
}

\begin{abstract}
Resumo
Este estudo tem como objetivo a verificação da consistência interna e estrutura fatorial da escala EMRI avaliadora da empatia em amostra de secundaristas e universitários no estado de Minas Gerais. Os instrumentos que mensuram a empatia podem ser encontrados com facilidade, porém, dos existentes, apenas a escala multidimensional de reatividade interpessoal (EMRI) é a que tem estrutura teórica e de medida de melhor organização, sendo a mais utilizada para avaliar a emaptia. Participaram do estudo 488 sujeitos, do sexo masculino e do sexo feminino, de 14 a 54 anos, distribuídos no nível fundamental e universitários da cidade de Patrocínio-MG. Os sujeitos responderam a Escala Multidimensional de Reatividade Interpessoal e dados sócio-demográficos. A partir de uma analise de equação e modelagem estrutural, observaram-se indicadores psicométricos que garantiram a consistência estrutural da escala, promovendo uma segurança da mensuração teórica do construto da empatia.

Palavras-chave: fidedignidade; modelagem estrutural; empatia; jovens.
\end{abstract}

\section{RELIABILITY OF THE FACTOR STRUCTURE OF THE MULTIDIMENSIONAL SCALE OF INTERPERSONAL REACTIVITY (EMRI)}

\begin{abstract}
This study aims to check the internal consistency and factor structure evaluative of the empathy scale in a high school and college sample in the state of Minas Gerais. The instruments that measure empathy can be easily found, however, of the existing, just multidimensional scale of interpersonal reactivity (Emri) is the theoretical framework that has far more and better organized, and the scale that is most commonly used to assess this construct. Participated 488 subjects, male and female, with ages from 1454 years old, distributed in primary and college levels in Patrocínio-MG composed this study sample. The subjects answered the Multidimensional Scale of Interpersonal Reactivity and socio-demographic data. From an equation analysis and structural modeling were observed psychometric indicators that assured the structural consistency of the scale, promoting in the security of the measure theoretical construct of empathy.
\end{abstract}

Keywords: trust; structural modeling; empathy; youth. 


\title{
FIABILIDAD DE LA ESTRUCTURA FACTORIAL DE LA ESCALA MULTIDIMENSIONAL DE REACTIVIDAD INTERPERSONAL (EMRI)
}

\begin{abstract}
Resumen
Este estudio tiene como objetivo la verificación de la consistencia interna y estructura factorial de la escala EMRI evaluativa de la empatía en muestra de estudiantes de la secundaria y universitarios en el estado de Minas Gerais. Los instrumentos que mensuran la empatía pueden ser encontrados con facilidad, pero, de los existentes, sólo la Escala Multidimensional de Reactividad Interpersonal (EMRI) es la que tiene estructura teórica y de medida más organizada, siendo la escala que es más utilizada para evaluar de ese constructo. Participaron 488 sujetos, del sexo masculino y del sexo femenino, de 14 a 54 años, distribuidos en el nivel secundario y universitario de la ciudad de Patrocínio-MG han participado de este estudio. Los sujetos respondieron la Escala Multidimensional de Reactividad Interpersonal y datos socio-demográficos. A partir de una análisis de ecuación y modelación estructural, se observó indicadores psicométricos que garantizan la consistencia estructural de la escala, promovió una medida de seguridad de lo constructo teórico de la empírica.
\end{abstract}

Palabras clave: fiabilidad; modelación estructural; empatía; jóvenes.

\section{INTRODUÇÃO}

A empatia é um construto psicológico que se refere a uma disposição funcional das pessoas para as trocas de experiências expostas de maneira incondicional em relação a outra pessoa; este construto é definido como uma resposta afetiva de origem evolutiva da forma mais apropriada frente à situação do outro do que da própria pessoa em si (Davis 1983; Hoffman, 2000). Sendo assim, uma pessoa considerada empática, teoricamente, é capaz de experimentar as emoções e/ou ter pensamentos que, supostamente, a outra pessoa tem ou estaria experimentado. Assim, a pessoa empática adotaria o ponto de vista do outro, compreenderia suas motivações e necessidades e atribuiria atitudes e comportamentos ao outro com a função de prover ajuda, agregação, cuidado, justiça e solidariedade (Batson, Eklund, Chermok, Hoyt \& Ortiz, 2007; Batson, Tricia, Highberger \& Shaw, 1995; Davis 1983; Decety, 2005; Decety \& Jackson, 2004; Decety, Michalska \& Akitsuki, 2008; Enz \& Zoll, 2006; Hoffman, 2000; Mehrabian \& Epstein, 1972; Wispé, 1990).

No Brasil é possível encontrar instrumentos que avaliam a empatia; cada um com seu modelo metodológico e teórico busca mensurar a capacidade das pessoas se colocarem no lugar do outro (Siqueira; Barbosa; Alves, 1999; Ribeiro et. al., 2002; Primi; Bueno; Muniz, 2006; Woyciekoski, 2006; Gouveia, Guerra, Santos, Rivera \& Singelis, 2007; Bandeira; Costa; Del Prette; Del Prette; GerkCarneiro, 2000; Del Prette; Del Prette, 2005; Falcone et. al., 2008; Galvão; Camino; Gouveia; Formiga, 2010). Apesar dessa quantidade de instrumentos, 
ainda tem se considerado a escala de empatia desenvolvida por Davis (1983), conhecida como Escala Multidimensional de Reatividade Interpessoal (EMRI), como uma das medidas mais usadas e importante para mensurar este construto, principalmente, porque ela é a escala que possui um corpo teórico organizado e de indicadores psicométricos que garantem uma definição e relação item-fator de forma consistente na abordagem do construto (Formiga, Rique, Galvão, Camino \& Mathias, 2011).

De acordo com Formiga e cols. (2011), bem como, Ribeiro et al. (2002), a EMRI tem sua importância, justamente, porque o autor da escala (Davis, 1983) desenvolveu uma perspectiva metodológica e teórica para uma medida da empatia nas pessoas, de forma que salienta construtos correlacionados a uma visão psicogenética, evolutiva e multidimensional da empatia; a importância de tal instrumento é assim tão grande, porque ao pesquisar nos sites de busca, nos últimos cinco anos, que dos 101 trabalhos empíricos encontrados, indexados ao PsycINFO, usaram a EMRI, versus 8 que utilizaram o Questionnaire Measure of Emotional Empathy (QMEE - questionário de emoção empática; Mehrabian \& Epstein, 1972) e 7 que usaram o Hogan Empathy Scale (HES - escala de empatia de Hogan, 1969) (Formiga, Galvão, Barboza \& Camino, 2012).

Com isso, a perspectiva teórica abordada na escala desenvolvida por Davis (1983) pressupõe que as habilidades empáticas se organizam em quatro construtos independentes, porém, não impedindo que estes possam se relacionar; esses construtos avaliam as experiências afetivas e cognitivas da pessoa: no que se refere à experiência cognitiva, destaca-se o construto tomada de perspectiva do outro (refere-se à capacidade cognitiva voltada para a compreensão e coordenação de percepções do outro que visem à solução de conflitos interpessoais e sociais) e fantasia (refere-se a habilidade de se identificar com personagens ficcionais em novelas, filmes e romances e sentir junto com eles, uma adesão involuntária às condições afetivas de alegria, tristeza, raiva etc. e/ou de necessidade destes personagens); em relação a experiência afetiva, esta, poderá ser acessada na pessoas através da consideração empática (diz respeito à capacidade de avaliar e sentir com o outro, bem como do reconhecer seus afetos e necessidades, que pode ser experimentada no self como uma motivação de cunho pró-social que pode levar ao comportamento de ajuda) e a angustia pessoal (refere-se a um sentimento de 
tensão e desconforto, frente à condição de necessidade do outro, podendo gerar comportamentos de afastamento ao invés de comportamentos de ajuda).

Esta escala tem sido traduzida e validada em muitas línguas, considerando tanto as dificuldades linguísticas e culturais. (Cliffordson, 2001; DeCorte, Buysse, Verhofstadt, Roeyers ponnet \& Davis, 2007; Escrivá, 2004; Kazmierczak, Plopa \& Retowski, 2007; Limpo, Alves \& Catro, 2010; Ribeiro, Koller \& Camino, 2001; Sampaio, Guimarães, Camino, Formiga \& Menezes, 2011; Siu \& Shek, 2005). Estudos desenvolvidos por Sampaio, Guimarães, Camino, Formiga e Menezes (2011), com amostras de jovens brasileiros de 17 a 25 anos de idade, confirmou a existência de quatro dimensões do construto da EMRI, observando indicadores psicométricos que garantiram a validade e consistência interna da estrutura fatorial. Formiga, Sampaio, Guimarães e Camino (2012), com o objetivo de não deixar dúvidas, pretenderam tornar acurado o resultado observado por Sampaio e cols. (2011). Assim, Formiga e cols. (2012), a partir de uma análise fatorial confirmatória, encontraram indicadores psicométricos que corroboraram a estrutura tetrafatorial previamente encontrada. Com isso, não somente se comprova a perspectiva teórica defendida por Davis (1983), mas, também, a organização fatorial, apontada por ele quando se pretender avaliar a empatia nas pessoas.

Sendo assim, o presente estudo tem como objetivo verificar a fidedignidade da estrutura fatorial apresentada pelos autores supracitados em uma amostra de sujeitos escolares e universitários do estado Minas Gerais. Ao enfatizar a fidedignidade de um teste, esta, refere-se à característica que ele deve possuir, na qual ao mensurar o fenômeno estudado com os mesmos sujeitos ou em outros em diferentes ocasiões garantir a precisão instrumental com um coeficiente próximo a 1.00; para isso pretende utilizar o Alfa de Cronbach como critério de consistência psicométrico (Kerlinger, 1980; Richardson, 1999; Cronbach, 1990; Anastasi \& Urbina, 2000).

O Alfa de Cronbach (a) é um dos indicadores psicométricos mais utilizados para verificar a fidedignidade ou validade interna do instrumento, o qual deverá apresentar um alfa acima de 0.70 ou igual a 1.00; desta maneira, quanto mais próximo desse número, melhor será a sua precisão; isto significa que os itens são homogêneos em sua medida, produzindo variância semelhante nesta, caracterizando uma segurança para a medida do fenômeno que se quer avaliar (Kline, 1994; Pasquali, 2001; Tabachnick \& Fidel, 1996). Sendo assim, espera-se 
encontrar uma estrutura fatorial semelhante a já encontrada pelos autores supracitados.

Terá, também, como contribuição para avaliação do estudo, a realização de uma análise fatorial confirmatória; estas técnicas levam em conta a teoria para definir os itens pertencentes a cada fator, bem como, apresentar indicadores de bondade de ajuste que permitem decidir objetivamente sobre a consistência e fidedignidade) do construto avaliado. Além de buscar a garantia da estimativa da magnitude dos efeitos estabelecidos entre variáveis, as quais estão condicionadas ao fato de o modelo especificado (isto é, o diagrama) estar correto e procurar corroborá-lo, pretende-se também, testar, se o modelo é consistente com os dados observados, a partir dos indicadores estatísticos, condição que permite dizer que resultado, modelo e dados são plausíveis, embora não se possa afirmar que este é correto (Farias \& Santos, 2000). Atende-se assim, não a certeza total do modelo, mas, a sua probabilidade sistemática na relação entre as variáveis e a perspectiva teórica assumida.

\section{MÉTODO}

\section{Participantes}

Participaram do estudo 488 sujeitos, do sexo masculino e do sexo feminino (57\%), de 14 a 54 anos $(M=19,02 ;$ d.p. = 6,03), distribuídos no nível fundamental (53\%) e universitários (47\%) da cidade de Patrocínio-MG. A amostra foi não probabilística, pois considerou-se a pessoa que, consultada, se dispusera a colaborar, respondendo o questionário que foi apresentado.

\section{Instrumentos}

\section{Escala Multidimensional de Reatividade Interpessoal de Davis - EMRI}

Trata-se de um instrumento elaborado por Davis (1983) e adaptado em sua versão original por Sampaio, Guimarães, Camino, Formiga e Menezes (2011) para o contexto brasileiro e corroborado, com suas quatro dimensões, no estudo de Formiga, Sampaio, Guimarães e Camino (2012). O instrumento é composto por 26 sentenças que descrevem comportamentos, sentimentos e características relacionadas à empatia, que são utilizadas para avaliar as seguintes dimensões da empatia: 
- Angústia pessoal (AP) - avalia as sensações afetivas de desconforto, incômodo e desprazer dirigidas para o self, quando o indivíduo imagina o sofrimento de outrem (por exemplo: Perco o controle quando vejo alguém que esteja precisando de muita ajuda; Fico apreensivo em situações emergenciais, etc.);

- Consideração empática (CE) - esta dimensão relaciona-se aos sentimentos dirigidos ao outro e à motivação para ajudar pessoas em necessidade, perigo ou desvantagem (Ex: Sinto compaixão quando alguém é tratado injustamente; Quando vejo que se aproveitam de alguém, sinto necessidade de protegê-lo, etc.);

- Tomada de perspectiva (TP) - mede a capacidade cognitiva do indivíduo de se colocar no lugar de outras pessoas, reconhecendo e inferindo o que elas pensam e sentem (Ex: Imagino como as pessoas se sentem quando eu as critico; Tento compreender meus amigos imaginando como eles vêem as coisas, etc.);

- Fantasia (FS) - a primeira designa a habilidade de se colocar no lugar de outras pessoas, tomando suas perspectivas e imaginando o que elas pensam ou sentem; a subescala de fantasia avalia a tendência de transpor a si mesmo imaginativamente, colocando-se no lugar de personagens de filmes e/ ou livros (Ex: Tenho facilidade de assumir a posição de um personagem do filme; Depois de ver uma peça de teatro ou um filme sinto-me envolvido com seus personagens, etc.).

Cada uma destas subescalas é composta, por uma quantidade específica de itens: FS e CE, sete proposições, AP e TP, seis proposições. Todas elas foram avaliadas por escalas likert, que variam de 1 ("não me descreve bem") a 5 ("descreve-me muito bem"). Escores mais altos indicam níveis mais elevados em cada uma dessas dimensões e a soma dos escores de todas as subescalas é utilizada para calcular o nível global de empatia. 0 item 2 (Sou neutro quando vejo filmes) deve ter sua pontuação invertida, pois foi elabo $\neg$ rado na direção contrária a dos demais itens da escala.

Além do EMRI foi utilizado um pequeno questionário para levantar alguns dados sociodemográficos como idade, sexo e renda econômica dos participantes. 


\section{Procedimentos}

Todos os procedimentos adotados nesta pesquisa seguiram as orientações previstas na Resolução 196/96 do CNS e na Resolução 016/2000 do Conselho Federal de Psicologia (CNS, 1996; ANPEPP, 2000).

Quatro colaboradores com experiência prévia na administração do EMRI foram responsabilizados pela coleta dos dados, e apresentaram-se nas salas de aula como interessados em conhecer as opiniões e os comportamentos dos alunos sobre as situações descritas nos instrumentos.

Solicitou-se a colaboração voluntária dos jovens no sentido de responderem um breve questionário. Após ficarem cientes das condições de participação na pesquisa, os sujeitos assinaram um termo de Consentimento Livre e Esclarecido. Foi-Ihes dito que não havia resposta certa ou errada. A todos foi assegurado o anonimato das suas respostas informando que estas seriam tratadas em seu conjunto. A Escala Multidimensional de Reatividade Interpessoal de Davis - EMRI foi respondida individualmente.

Apesar de o instrumento ser auto-aplicável, contando com as instruções necessárias para que possam ser respondidos, os colaboradores na aplicação estiveram presentes durante toda a aplicação para retirar eventuais dúvidas ou realizar esclarecimentos que se fizessem indispensáveis. Um tempo médio de 30 minutos foi suficiente para concluir essa atividade.

Quanto à análise dos dados, tomou-se orientação metodológica e estatística o estudo de Sampaio, Guimarães, Camino, Formiga e Menezes (2011) e Formiga, Sampaio, Guimarães e Camino (2012); na versão 18.0 do pacote estatístico SPSS para Windows, foram computadas estatísticas descritivas (tendência central e dispersão) e cálculo referido ao Alpha de Cronbach (a). Realizou-se, no AMOS GRAFICS 18.0, a análise fatorial confirmatória, esta tinha o objetivo de avaliar o modelo multidimensional, previamente encontrado por esses autores, bem como, os indicadores psicométricos da estrutura fatorial proposta.

Considerou-se como entrada a matriz de covariâncias, tendo sido adotado o estimador ML (Maximum Likelihood). Sendo um tipo de análise estatística mais criteriosa e rigorosa, testou-se a estrutura teórica que se propõe neste estudo: isto é, a estrutura com quatro fatores. Esta análise apresenta alguns índices que permitem avaliar a qualidade de ajuste do modelo proposto (Bilich, Silva \& Ramos, 2006; Byrne, 1989; Hair, Tatham, Anderson \& Black, 2005; Tabachnick 
\& Fidell, 1996; Van De Vijver \& Leung, 1997). A seguir serão apresentados esses indicadores:

- O $\mathrm{X}^{2}$ (qui-quadrado) testa a probabilidade do modelo teórico se ajustar aos dados: quanto maior o valor do $\mathrm{X}^{2}$ pior o ajustamento. Entretanto, ele tem sido pouco empregado na literatura, sendo mais comum considerar sua razão em relação aos graus de liberdade ( $x^{2} / g . I$.). Neste caso, valores até 3 indicam um ajustamento adequado.

- Raiz Quadrada Média Residual (RMR), que indica o ajustamento do modelo teórico aos dados, na medida em que a diferença entre os dois se aproxima de zero (Joreskög \& Sörbom, 1989).

- O Goodness-of-Fit Index (GFI) e o Adjusted Goodness-of-Fit Index (AGFI) são análogos ao $\mathrm{R}^{2}$ na regressão múltipla e, portanto, indicam a proporção de variância-covariância nos dados explicada pelo modelo. Os valores desses indicadores variam de 0 a 1 , sendo que os valores na casa dos 0,80 e 0,90, ou superiores, indicam um ajustamento satisfatório (Hair, Anderson, Tatham \& Black, 2005; Bilich, Silva, Ramos, 2006).

- A Root-Mean-Square Error of Approximation (RMSEA), com seu intervalo de confiança de 90\% (IC90\%), é considerado um indicador de "maldade" de ajuste, isto é, valores altos indicam um modelo não ajustado. Assume-se como ideal que o RMSEA se situe entre 0,05 e 0,08, aceitando-se valores até 0,10 (Hair, Anderson, Tatham \& Black, 2005).

- O Comparative Fit Index (CFI) compara de forma geral o modelo estimado ao modelo nulo, considerando valores mais próximos de um como indicadores de ajustamento satisfatório (Hair, Tatham, Anderson \& Black, 2005; Bilich, Silva \& Ramos, 2006).

- Tucker-Lewis Index (TLI), apresenta uma medida de parcimônia entre os índices do modelo proposto e do modelo nulo. Varia de zero a um, com índice aceitável acima de 0,90 (Bilich, Silva \& Ramos, 2006).

- O Expected Cross-Validation Index (ECVI) e o Consistent Akaike Information Criterion (CAIC) são indicadores geralmente empregados para avaliar a adequação de um modelo determinado em relação a outro. Valores baixos do ECVI e CAIC expressam o modelo com melhor ajuste (Hair, Anderson, Tatham \& Black, 2005; Bilich, Silva, Ramos, 2006). 


\section{RESULTADOS E DISCUSSÃO}

A fim de atender o objetivo principal do presente estudo, empregou-se o pacote estatístico AMOS 18.0 para efetuar uma análise fatorial confirmatória. Testou-se assim, o modelo proposto por Davis (1983), adaptado por Sampaio et. al. (2011) e corroborado por Formiga et al. (2012) para o contexto brasileiro; de acordo com os autores supracitados, a estrutura multidimensional de reatividade interpessoal contempla quatro fatores. Para comprovar a estrutura proposta optou-se por deixar livre as covariâncias (phi, $\varphi$ ) entre os fatores, revelando que os indicadores de qualidade de ajuste para cada modelo se mostraram próximos as recomendações apresentadas na literatura (Byrne, 1989; Tabachnick \& Fidell, 1996; Van De Vijver \& Leung, 1997).

De acordo com os resultados obtidos nas análises (ver Tabela 1), o modelo tetrafatorial, já proposto e observado pelos autores supracitados apresentarem indicadores estatísticos que justificam tanto a sua fidedignidade estrutural quanto a acurácia desse modelo em diferentes amostras. Seja na amostra geral ou nas amostras de cada amostra separada (isto é, amostra de escolares e de universitários), os indicadores revelaram-se próximos aos exigidos pela literatura estatística sobre a equação e modelagem estrutural, garantindo com isso, a confirmação teórico-empírica do construto avaliado.

Tabela 1.

Indicadores psicométricos da estrutura fatorial da Escala Multidimensional de Reatividade Interpessoal (EMRI) de Davis em jovens de Minas Gerais.

\begin{tabular}{cccccccccc}
\hline Amostras & $X^{2} / g l$ & RMR & GFI & AGFI & CFI & TLI & $\begin{array}{c}\text { RMSEA } \\
\text { (intervalo) }\end{array}$ & CAIC & $\begin{array}{c}\text { ECVI } \\
\text { (intervalo) }\end{array}$ \\
\hline Amostra 1 & 1,02 & 0,05 & 0,97 & 0,95 & 1,00 & 0,99 & $\begin{array}{c}0,01 \\
(0,01-0,02)\end{array}$ & 1444,67 & $\begin{array}{c}1,09 \\
(1,08-1,16)\end{array}$ \\
Amostra 2 $^{\text {b }}$ & 1,16 & 0,06 & 0,96 & 0,93 & 0,99 & 0,99 & $\begin{array}{c}0,03 \\
(0,01-0,05)\end{array}$ & 1038,71 & $\begin{array}{c}2,06 \\
(2,03-2,21)\end{array}$ \\
Amostra 3 & 1,23 & 0,06 & 0,95 & 0,92 & 0,99 & 0,98 & $\begin{array}{c}0,03 \\
(0,01-0,04)\end{array}$ & 1287,57 & $\begin{array}{c}2,17 \\
(2,03-2,34)\end{array}$ \\
\hline
\end{tabular}

Notas: $a=$ Amostra total; $b=$ Amostra escolares; $c=$ Amostra universitários.

Com todas as saturações (Lambdas, $\lambda$ ) dentro do intervalo esperado (0-1), denotou-se não haver problemas de estimação proposta, tendo todas elas sido estatisticamente diferentes de zero $(t>1,96 ; p<0,05)$. Tais resultados corroboram que a estrutura psicométrica composta por quatro fatores 
[Consideração Empática (CE), Angústia Pessoal (AP) e Tomada de Perspectiva (TP) e Fantasia (FS)] avaliam a empatia assumida pelos sujeitos. Estes fatores, por sua vez, apresentaram lambdas $(\lambda)$ associativos positivos entre si que estiveram dentro do intervalo esperado: na amostra total os lambdas foram de 0,82 a 0,91, na amostra de escolares de 0,81 a 0,91, na amostra de universitários de 0,77 a 0,92 .

Para não deixar duvidas da consistência da escala EMRI, efetuou-se uma calculo do alfa de Cronbach para os fatores; estes variaram na amostra total e na amostra de escolares e universitários alfas os seguintes: Consideração Empática $(C E)=0,79$ para os escolares e 0,83 para universitários; Angústia Pessoal $(A P)=0,72$ para os escolares e 0,77 para os universitários; Tomada de Perspectiva $(\mathrm{TP})=0,74$ para os escolares e 0.80 para universitários; Fantasia $(\mathrm{FS})]=0,77$ para os escolares e 0,78 para universitários. Tais resultados indicam uma consistência interna dos fatores, garantindo com isso, a mensuração destes fatores nas amostras avaliadas em distintos sujeitos.

A partir desses resultados, a EMRI composta por quatro fatores, apresentou uma adequabilidade e consistência interna de sua medida, independente do contexto amostral administrado. Comparando os resultados observados com os estudos de Sampaio, Guimarães, Camino, Formiga e Menezes (2011), bem como, de Formiga, Sampaio, Guimarães e Camino (2012) em amostras brasileiras, os indicadores psicométricos da estrutural fatorial estão próximos, tanto aos indicadores que esses autores encontraram quanto ao que é exigido na literatura clássica sobre o calculo (Byrne, 1989; Tabachnick \& Fidell, 1996; Van De Vijver \& Leung, 1997) tornando a EMRI fidedigna estrutural e funcionalmente na sua mensuração.

Com isso, é possível salientar que a estrutura fatorial da EMRI responde não somente a condição da medida da empatia em brasileiros, mas, também, da direção teórica que Davis (1983) apresentou quanto ao construto avaliativo da empatia comprovando as reflexões teóricas e empíricas dos estudos brasileiros para avaliação desse construto (ver Sampaio, Guimarães, Camino, Formiga \& Menezes, 2011; Formiga, Sampaio, Guimarães \& Camino, 2012). A medida da empatia, a partir da EMRI, revela probabilidade do sujeito gerar uma capacidade em desenvolver o reconhecimento de uma situação e a preocupação com o outro, o qual trata-se de uma espécie de ressonância interpessoal, que, será 
manipulada através das experiências empáticas vividas pela pessoa por meio do afeto e/ou da cognição (Formiga, 2012).

Partindo da reflexão no paragrafo acima, pode-se apontar em direção de que a pessoa empática, ao buscar o respeito, a compreensão do outro e a participação no espaço sócio-cognitivo do observador no campo das experiências conflituosas do outro, terá, antes, que estar disposto às aberturas do espaço interpessoal em pensamento e afeto, o qual, provavelmente, estimulará e/ou simulará convicções, desejos, percepções, sentimento, etc. que permita ao sujeito se colocar no lugar do sentimento e emoção do outro, mesmo não sendo o outro. (Formiga, 2012; Formiga, Sampaio, Guimarães \& Camino, 2012). É possível observar tal concepção quanto se observa não somente os resultados dos alfas, o qual segure a consistência da escala, mas, as associações lambdas $(\square)$ positivas entre os fatores [por exemplo, Consideração Empática (CE), Angústia Pessoal (AP) e Tomada de Perspectiva (TP) e Fantasia (FS)], os quais, sugere a interdependência entre eles; isto é, ao estimular um desses fatores, possivelmente, os demais serão estimulados.

De forma geral, os estudos sobre o construto da empatia e, especificamente, a avaliação das EMRI, permite compreender que as pessoas têm a capacidade de abrir canais de comunicação para a relação interpessoal de trocas de ações cooperativas. É possível então, que a partir do desenvolvimento dessas habilidades venha a se estabelecer condições para uma formação moral, ética e de direitos humanos (Formiga, Camino \& Galvão, 2009; Camino \& Camino, 1996; Eisenberg \& Miller, 1987; Hoffman, 2000; Sampaio, Monte, Camino \& Roazzi, 2008); pensar nessa direção se deve, porque, esses construtos têm em comum a busca do respeito e compreensão da alteridade e a inclusão do outro (observador ou observado) no campo do problema, possibilitando a quem precisa de ajuda e a quem pode ajudar uma disposição para o acolhimento e apoio dos vínculos afetivos na interação humana (Formiga et al, 2011).

Por fim, espera-se que os objetivos deste estudo tenham sido cumpridos, principalmente, no que diz respeito à consistência interna da estrutura fatorial da escala EMRI; esta, por sua vez, poderá ser administrada a sujeitos brasileiros para estudos na área das ciências humanas e sociais. Todavia, é bom destacar que quando considerar os resultados do presente estudo em outros contextos sociais é necessário ter em conta os aspectos mais específicos ou universais de cada cultura na avaliação dessas escalas quando se pretender refutá-las ou não 
com outras amostras considerando o espaço geo-político e social (Muenjohn \& Armstrong, 2007; Triandis e cols, 1993; Triandis, 1994; Van de Vijver \& Leung, 1997).

Apesar da consistência dos resultados neste estudo, salienta-se áreas de estudos mais específicos, por exemplo: avaliar a condição desenvolvimentista das estrutural fatorial da EMRI (fazendo referência a idade e classe social) na dinâmica interna da família, aplicando o mesmo instrumento em pais e filhos; não menos importante, seria útil, reunir evidências da validade e precisão intra, inter e pan-cultural, a fim de avaliar sua validade de critério, convergente e/ou divergente, bem como, conhecer a estabilidade temporal (teste-reteste) e política na avaliação do construto da empatia; outro estudo, também lucrativo, seria o da relação entre empatia e reconhecimento da emoção em diferentes contextos sócio-religiosos.

\section{REFERÊNCIAS}

Anastasi, A. \& Urbina, S. (2000). Fidedignidade. Em: Testagem psicológica. (pp. 84-105). Artmed: Porto Alegre.

Associação Nacional de Pesquisa e Pós-Graduação em Psicologia - ANPEPP. (2000). Contribuições para a discussão das Resoluções CNS no. 196/96 e CFP No 016/2000. Recuperado em 02 de Setembro de 2011, de: http://www.anpepp.org.br/XIISimposio/Rel_ComissaoEticasobre_Res_CNS_ e_CFP.pdf2000.

Bandeira, M., Costa, M. N., Del Prette, Z. A. P., Del Prette, A. \& Gerk-Carneiro, E. (2000). Qualidades psicométricas do Inventário de Habilidades Sociais (IHS): Estudo sobre a estabilidade temporal e a validade concomitante. Estudos de Psicologia, 5(2), 401-412.

Batson, C. D., Eklund, J. H., Chermok, V. L., Hoyt, J. L. \& Ortiz, B. G. (2007). An additional antecedent of empathic concern: Valuing the welfare of the person in need. Journal of Personality and Social Psychology, 93(1), 65-74.

Batson, D. C., Tricia, R. K., Highberger, L. \& Shaw, L. L. (1995). Immorality from empathy-induced altruism: When compassion and justice conflict. Journal of Personality and Social Psychology, 68(6), 1042-1054.

Bilich, F., Silva, R. \& Ramos, P. (2006). Análise de flexibilidade em economia da informação: Modelagem de equações estruturais. Revista de Gestão da Tecnologia e Sistemas de Informação, 3(2), 93-122. 
Byrne, B. M. (1989). A primer of LISREL: Basic applications and programming for confirmatory factor analytic models. New York: Springer-Verlag.

Camino, C. \& Camino, L. (1996). Julgamento moral, emoção e empatia. In Z. D. Trindade \& C. Camino (Eds.), Cognição social e juízo moral (Coletâneas da ANPEPP), (pp. 109-135). Rio de Janeiro: Associação Nacional de Pesquisa e Pós-graduação em Psicologia.

Cliffordson, C. (2001). Parent's judgments and student's self-judgments of empathy. European Journal of Psychological Assessment, 17, 36-47.

Conselho Nacional De Saúde - CNS. (1996). Diretrizes e normas regulamentadoras de pesquisas envolvendo seres humanos. Recuperado em 02 de Setembro de 2011, de: http://conselho.saude.gov.br/resolucoes/reso_96.htm. 1996.

Cronbach, L. (1990). Como julgar os testes: Fidedignidade e outras qualidades. Em: Fundamentos da testagem psicológica. (pp. 176-197). Artes médicas: Porto Alegre.

Davis, M. H. (1983). Measuring individual differences in empathy: Evidence for a multidimensional approach. Journal of Personality and Social Psychology, 44, 113-126.

Decety, J., Michalska, K. J. \& Akitsuki, Y. (2008). Who caused the pain? A functional MRI investigation of empathy and intentionality in children. Neuropsychologia, 46, 2607-2614.

Decety, J. (2005). Perspective taking as the royal avenue to empathy. In: B. F. Malle e S. D. Hodges (Eds.), Other minds: How humans bridge the divide between self and other. (pp. 143-157). New York: Guilford Publications.

Decety, J. \& Jackson, P. L. (2004). The functional architecture of human empathy. Behavioral and Cognitive Neuroscience Reviews, 3, 71-100.

DeCorte, K., Buysee, A., Verhofstadt, L., Roeyers, H., Ponnet, K. \& Davis, M. (2007). Measuring empathic tendencies: Reliability and validity of the Dutch version of the Interpersonal Reactivity Index. Psichologica Belgica, 47(4), 235-260.

Del Prette, Z. A. P. \& Del Prette, A. (2005). Psicologia das habilidades sociais na infância: Teoria e prática. Petrópolis, RJ: Vozes.

Eisenberg, N. \& Miller, P. A. (1987). The relation of empathy to prosocial and related behaviors. Psychological Bulletin, 101, 91-119. 
Enz, N. \& Zoll, N. (2006). Cultural differences in empathy between China, Germany and the UK. Recuperado em 23 de novembro de 2006, de www.nicve.salford.ac.uk/elvis/ resources/empathy.

Escrivã, V. M. (2004). Measuring empathy: The Interpersonal Reactivity Index. Psicothema, 16(2), 255-260.

Falcone, E. M. O., Ferreira, M. C., Luz, R. C. M., Fernandes, C. S., Faria, C. A., D'augustin, J. F., Sardinha, A. \& Pinho, V. D. (2008). Inventário de Empatia (I.E.): Desenvolvimento e validação de uma medida brasileira. Avaliação Psicológica, 7(3), 321-334.

Farias, S. A. \& Santos, R. C. (2000). Modelagem de equações estruturais e satisfação do consumidor: Uma investigação teórica e prática. Revista de Administração Contemporânea, 4(3), 107-132.

Formiga, N. S.; Camino, C. \& Galvão, L. (2009). Empatia, desenvolvimento moral e conduta desviante em adolescentes: Testagem de um modelo teórico. In: VII Congresso Brasileiro de Psicologia do Desenvolvimento. (pp. 541-542). Rio de Janeiro, RJ: CBPD.

Formiga, N. S. (2012). Os estudos sobre empatia: Reflexões sobre um construto psicológico em diversas áreas científicas. Revista Eletrônica psicologia.com.pt - O Portal dos Psicólogos, 1, 1-25. Recuperado em 10 de Novembro de 2012,

de: http://www.psicologia.pt/artigos/ver_artigo.php?codigo=A0639

Formiga, N. S.; Galvão, L. K. S; Barboza, M. \& Camino, C. (2012). Consistência estrutural da escala multidimensional de reatividade interpessoal: Um estudo com jovens civis e militares. Artigo submetido para avaliação.

Formiga, N., Rique, J., Galvão, L., Camino, C. \& Mathias, A. (2011). Escala Multidimensional de Reatividade Interpessoal - EMRI: Consistência estrutural da versal reduzida. Revista Psicologia, Trujillo (Perú), 13(2), 188198.

Galvao, L., Camino, C., Gouveia, V. V. \& Formiga, N. S. (2010). Proposta de uma medida de empatia focada em grupos: Validade fatorial e consistência interna. Psico, 41(3), 399-405.

Gouveia, V. V., Guerra, V. M., Santos, W. S., Rivera, G. A. \& Singelis, T. M. (2007). Escala de Contágio Emocional: Adaptação ao contexto brasileiro. Psico, 38(1), 45-54. 
Hair, J. F., Tatham, R. L., Anderson, R. E. \& Black, W. (2005). Análise multivariada de dados. Porto Alegre: Bookman.

Hoffman, M. L. (2000). Empathy and moral development: Implications for caring and justice. New York: Cambridge University Press.

Hogan, R. (1969). Development of an empathy scale. Journal of Consulting and Clinical Psychology, 33, 307-316.

Joreskög, K. \& Sörbom, D. (1989). LISREL 7 user's reference guide. Mooresville: Scientific Software.

Limpo, T., Alves, R. A. \& Catro, S. L. (2010). Medir a empatia: Adaptação portuguesa do índice de reactividade interpessoal. Laboratório de Psicologia, $8(2), 171-184$.

Kazmierczak, M., Plopa, M. \& Petowski, S. (2007). Empathic sensitiveness scale. Przeglad Psychoogiczny, 50(1), 9-24.

Kerlinger, F. N. (1980). Metodologia da pesquisa em ciências sociais. São Paulo: EPU.

Kline, P. (1994). An easy guide to factor analysis. Routledge: New York, NY.

Pasquali, L. (2001). Técnicas de exame psicológico - TEP. Manual de fundamentos das técnicas psicológicas. São Paulo: Casa do psicólogo.

Primi, R., Bueno, J. M. H. \& Muniz, M. (2006). Inteligência emocional: Validade convergente e discriminante do MSCEIT com a BPR-5 e o 16PF. Psicologia: Ciencia e Profissão, 26(1), 26-45.

Mehrabian, A. \& Epstein, N. (1972). A measure of emotional empathy. Journal of Personality, 40, 525-543.

Muenjohn, N. \& Armstrong, A. (2007). Transformational Leadership: The Influence of Culture on the Leadership Behaviours of Expatriate Managers. International Journal of Business and Information, 2(2), 265-283.

Ribeiro, J., Koller, S. H. \& Camino, C. (2002). Adaptação e validação de duas escalas de empatia para uso no Brasil. Estudos de Psicologia, 18(3), 43-53.

Richardson, R. J. (1999). Pesquisa social: Métodos e técnicas. São Paulo: Atlas.

Sampaio, L. R., Guimarães, P. R. B., Camino, C. P. S., Formiga, N. S. \& Menezes, I. G. (2011). Estudos sobre a dimensionalidade da empatia: Tradução e adaptação do Interpersonal Reactivity Index (IRI). Psico, 42(1), 67-76.

Sampaio, L. R., Monte, F. C., Camino, C. \& Roazzi, A. (2008). Justiça distributiva e empatia em adolescentes do nordeste brasileiro. Psicologia: Reflexão e Crítica, 21(2), 275-282. 
Siqueira, M. M.; Barbosa, N. C. \& Alves, M. T. (1999). Construção e validação fatorial de uma medida de inteligência emocional. Psicologia: Teoria e Pesquisa, 15(2), 143-152.

Siu, A. M. H., \& Shek, D. T. L. (2005). Validation of the Interpersonal Reactivity Index in a Chinese context. Research on Social Work Practice, 15(2), 118126.

Tabachnick, B. G. \& Fidell, L. S. (1996). Using multivariate statistics. Needham Heights, MA: Allyn \& Bacon.

Triandis, H.C. (1995). Individualism and collectivism. Boulder, CO: Westview Press.

Trianis, H. C. e cols. (1993). An etic-emic analysis of individualism and collectivims. Journal of Cross-cultural Psychology, 24(3), 366-383.

Van De Vijver, F. \& Leung, K. (1997). Methods and data analysis for crosscultural research. Thousand Oaks, CA: Sage Publications.

Wispé, L. (1990). History of the concept of empathy. In: N. Eisenberg \& J. Strayer (org), Empathy and its development. (pp 17-37). New York: Cambridge University Press.

Contato: nsformiga@yahoo.com

Recebido em: 21/05/2013

Revisado em: 23/06/2013

Aceito em: 09/08/2013 